University of Nebraska - Lincoln

DigitalCommons@University of Nebraska - Lincoln

Faculty Publications, Department of Psychology

Psychology, Department of

August 1998

\title{
Methodological Considerations in Pretrial Publicity Research: Is the Medium the Message?
}

Jeffrey R. Wilson

Louisiana State University,

Brian H. Bornstein

University of Nebraska-Lincoln, bbornstein2@unl.edu

Follow this and additional works at: https://digitalcommons.unl.edu/psychfacpub

Part of the Psychiatry and Psychology Commons

Wilson, Jeffrey R. and Bornstein, Brian H., "Methodological Considerations in Pretrial Publicity Research: Is the Medium the Message?" (1998). Faculty Publications, Department of Psychology. 172.

https://digitalcommons.unl.edu/psychfacpub/172

This Article is brought to you for free and open access by the Psychology, Department of at DigitalCommons@University of Nebraska - Lincoln. It has been accepted for inclusion in Faculty Publications, Department of Psychology by an authorized administrator of DigitalCommons@University of Nebraska - Lincoln. 


\title{
Methodological Considerations in Pretrial Publicity Research: Is the Medium the Message?
}

\author{
Jeffrey R. Wilson and Brian H. Bornstein \\ Department of Psychology, Louisiana State University, Baton Rouge
}

Two methodological issues within the pretrial publicity (PTP) literature were examined in the present experiment: the effect of emotional versus factual PTP and the effect of presenting PTP through different media. Emotional and factual PTP were constructed that differed in level of emotionality, but produced the same degree of bias. The PTP was presented in either a videotaped or written format. Although there was a significantly biasing effect of PTP overall compared to a control condition, no significant difference was found either between factual and emotional PTP or between video and written PTP.

\section{INTRODUCTION}

Two of the guarantees of our constitution are frequently viewed as coming into conflict with one another: the freedom of the press and the right to a fair trial (Carroll, Kerr, Alfini, Weaver, MacCoun, \& Feldman, 1986; Linz \& Penrod, 1992; Simon, 1980, Chapter 6). One of the arguments behind this conflict is that excessive pretrial publicity (PTP) about a case will prejudice potential jurors' judgments, thereby compromising a defendant's right to a fair trial. At the present time there are still no formal guidelines for determining when and how PTP affects jurors' decisions (Linz \& Penrod, 1992). Researchers have examined PTP in hopes of informing the courts of the impact of different types of PTP as well as judicial remedies for debiasing jurors who have been exposed to PTP. Researchers disagree about whether there is enough empirical support to help the courts in establishing guidelines for PTP (Carroll et al., 1986; Fulero, 1987).

Although not all studies have found a prejudicial effect of PTP (e.g., Davis, 1986), the majority of studies on PTP have established support for its prejudicial impact toward proprosecution verdicts by potential or mock jurors (Costantini \& King, 1980; Hoiberg \& Stires, 1973; Kramer, Kerr, \& Carroll, 1990; Moran \& Cutler, 1991; Ogloff \& Vidmar, 1994; Otto, Penrod, \& Dexter, 1994; PadawerSinger \& Barton, 1975; Simon \& Eimermann, 1971; Sue, Smith, \& Gilbert, 1974; Sue, Smith, \& Pedroza, 1975; Tans \& Chaffee, 1966). This lack of uniformity might reflect methodological variations in how research on PTP is conducted; researchers of PTP have operationalized it in a variety of ways and varied the means of presenting it. 


\section{Emotional versus Factual PTP}

A few studies have made a distinction between emotional and factual PTP (Hoiberg \& Stires, 1973; Kramer \& Kerr, 1989; Kramer et al., 1990). Factual PTP typically consists of unsensational information that would be relevant to jurors' decisions if admitted into evidence; that is, it raises potential jurors' subjective certainty in the defendant's guilt (e.g., hearing that the defendant confessed to the crime). Emotional PTP typically consists of sensationalized and lurid details about the case that may or may not be informative in an evidentiary sense, but that emotionally arouses potential jurors, thereby prejudicing them against the defendant (e.g., describing in detail the brutal rape of a young woman; Hoiberg \& Stires, 1973). Researchers typically assume that emotional and factual PTP differentially affect juror decision making: emotional PTP through emotional arousal, and factual PTP through the damaging information that it provides about the defendant (Kramer et al., 1990).

The results of these studies have yet to provide strong evidence as to the value of this distinction. For example, Hoiberg and Stires (1973) found that males were not affected by either factual or emotional PTP, whereas females were affected only by the emotional PTP Although it therefore appears that sex differences exist for emotional PTP, these results are misleading. Because the trial used by Hoiberg and Stires concerned the rape of a female, it is perhaps not surprising that females' decisions were more affected by lurid details of a rape than were males' decisions. The sex differences may have been more of an artifact in the study than some form of general difference in the way male and female jurors make decisions (Sue et al., 1975).

Kramer and Kerr (1989), on the other hand, found evidence that both emotional and factual PTP produced significant bias against the defendant. When either type of PTP was present, participants rendered significantly more guilty verdicts than when PTP was absent. The difference in results between the two studies can probably be attributed to the nature of the PTP stimuli. Kramer and Kerr (1989) went to considerable lengths to produce realistic news reports and newspaper articles. Also, participants were exposed to PTP anywhere from 3 to $14 \mathrm{~min}$, depending upon the condition. Hoiberg and Stires (1973) presented participants with just one page of written PTP for each of their conditions and gave participants 4 min to read the page.

Although both of these studies employed factual and emotional PTP, neither one compared factual PTP to emotional PTP directly. Therefore, it is unclear as to which type of PTP is more damaging, factual or emotional PTP This failure to compare factual and emotional PTP has led to several other problems concerning the distinction. First, the distinction implies that factual PTP does not produce emotional arousal, while it assumes that emotional PTP is emotionally arousing. Only one study has constructed and validated emotional PTP that is emotionally arousing and found factual PTP to be unarousing (Kramer et al., 1990). 
Second, most presentations of emotional and factual PTP vary in the duration and the amount of information they contain. Emotional PTP is typically longer and includes more details about an event than factual PTP (Kramer \& Kerr, 1989; Kramer et al., 1990), which may make emotional PTP more informative to a juror when making a judgment of guilt. Therefore, it may be spurious to associate emotional arousal alone with the effects of emotional PTP. The present research addresses these problems by holding the length of presentation, the number of PTP items, and the PTP's effect on participants' perceptions of guilt constant, while manipulating the level of emotional arousal produced by factual versus emotional PTP.

\section{Means of Presenting PTP}

Research on PTP is conducted through two different means. Some studies have used surveys to assess the effect of PTP on potential jurors' perception of the defendant (Costantini \& King, 1980; Moran \& Cutler, 1991, 1997; Simon \& Eimermann, 1971). Surveys try to assess PTP bias in natural settings, typically through telephoning potential jurors for upcoming trials that have received considerable publicity. In these types of studies, researchers examine whether there is a correlation between the amount of exposure to PTP and the perceived guilt of the defendant. Results from these studies generally find high positive correlations between the amount of exposure to PTP for a particular case and the perceived culpability of the defendant for that case (e.g., Moran \& Cutler, 1991, 1997). Thus, it appears that as potential jurors' knowledge increases about a case, they become more likely to perceive the defendant as guilty.

However, surveys are not without limitations. First, surveys rely on correlational data. Although one can discover relationships between variables using correlational data, correlations do not provide conclusive evidence that PTP causes differential verdicts. Second, surveys only indirectly test the relationship between PTP and juror decision making, because participants are not exposed to a trial, which could mitigate any effects of PTP (Otto et al., 1994).

The bulk of the studies on PTP are done using jury simulations. PTP simulations have been enacted in a variety of ways. Most studies have used written forms of PTP represented as newspaper articles (Davis, 1986; Hoiberg \& Stires, 1973; Otto et al., 1994; Padawer-Singer, Singer, \& Singer, 1977; Sue et al., 1974, 1975; Tans \& Chaffee, 1966), but videotaped PTP has also been used on occasion (Kramer et al., 1990; Ogloff \& Vidmar, 1994). Ogloff and Vidmar (1994) examined the effect on jurors' decisions of using different media to present PTP The PTP in their experiment was comprised of excerpts from real newspaper and television programs about a sexual molestation case at an orphanage. They found that jurors exposed to televised PTP gave significantly higher guilt ratings than jurors who read the PTP. Furthermore, jurors who read the PTP gave significantly higher guilt ratings than jurors who read a control article presenting basic facts about the case. 
Although these findings are impressive by virtue of Ogloff and Vidmar's (1994) use of an actual case and authentic PTP, this realism is also problematic. A necessary consequence of using authentic PTP was that the information in the television condition was not identical to the information in the reading condition, meaning that Ogloff and Vidmar (1994) did not merely manipulate the medium. Although they argued that the information was similar across conditions, this lack of constancy means that the particular medium itself may not have been the sole reason for increased bias. In addition, Ogloff and Vidmar did not expose their participants to a trial. Although it is not uncommon to examine the effects of PTP in the absence of a trial (e.g., Tans \& Chaffee, 1966), PTP in general has less bearing on jurors' decisions after they have seen and heard the trial evidence (Otto et al., 1994). The differential effect of presenting PTP through different media (Ogloff \& Vidmar, 1994) may diminish in the presence of a trial as well.

\section{Overview}

The purpose of the present experiment was to examine some of the methodological issues described above. First, no study has examined whether the medium of PTP will affect jurors' decisions after they have been exposed to a trial. A second goal of the present research was to examine further the distinction between emotional and factual PTP. No study has directly examined which type of PTP produces greater effects on judgments of guilt. A direct comparison between these two types of PTP would provide evidence as to which type of PTP is more damaging in biasing jurors' verdicts against the defendant.

Specifically, participants were exposed to either factual or emotional PTP in one of two ways: television report or written summary. Chaiken and Eagly $(1976,1983)$ have shown that videotape is a more persuasive medium than written material when the message is easy to understand. In support of these findings, Ogloff and Vidmar (1994) reported that videotaped PTP produced significantly greater prejudice toward the defendant than written PTP. However, as Ogloff and Vidmar did not expose participants to a trial, it is unclear whether their results would generalize to a more forensically valid situation where participants were shown a trial. Therefore, the present experiment extends Ogloff and Vidmar's results by asking whether there is an effect of PTP medium when participants actually see a trial and the content of the PTP is experimentally controlled so that it does not differ between media. Consistent with their findings, it is hypothesized that presenting PTP on television will produce significantly higher guilt ratings than a written summary of PTP.

Also, although no direct evidence exists on which type of PTP should produce greater bias against the defendant, most studies have shown some indirect support for a greater effect of emotional than of factual PTP (Hoiberg \& Stires, 1973; Kramer et al., 1990). Therefore, it is hypothesized that emotional PTP will produce higher estimates of guilt than factual PTP. Furthermore, both types of PTP should produce significantly higher estimates of guilt than a control condition with no PTP. 


\section{METHOD}

\section{Participants}

The participants were 88 undergraduates in psychology courses who received extra course credit.

\section{Design}

A $3 \times 2$ between-subjects design was used. Participants were randomly exposed to either control PTP, factual PTP, or emotional PTP through one of two media. The control PTP consisted of basic information concerning the case. Both the factual and emotional PTP consisted of basic information concerning the case, plus additional PTP items designed to elicit prejudice against the defendant. The emotional PTP was designed to be significantly more emotionally arousing than either the factual or the control PTP. In the "video" condition, participants watched a news reporter read their respective PTP on a television monitor. In the "written" condition, participants read a news article containing their respective PTP. The content of the PTP was identical across media.

\section{Materials}

An abridged videotaped copy of an actual trial, State of California v. Winters, was used for the experiment. The videotape concerns a woman who is on trial for the stabbing death of her mother. The major issue at trial is whether the defendant is guilty of murder or manslaughter (i.e., whether she stabbed her mother is not in dispute). On tape, the trial lasts approximately $2 \mathrm{hr}$. A 19-inch color television monitor was used to show the trial.

The control article was constructed using basic information about the case from the trial. The article also served as a written transcript for the control video. The control video included a middle-aged man dressed in a suit and overcoat standing outside a court house. The goal was to have the video as close to a newscast as possible. The man read cue cards that contained the information from the article. The man was directed to read the cards in a neutral tone at an average rate of speed. This insured that the medium was the only factor being manipulated between the video and written conditions. The same process was performed in constructing the emotional and factual PTP. All of the articles included information that was consistent with information presented at trial; however, both the emotional and factual PTP included eight additional negative items that were weighted toward the defendant being guilty of murder. The articles were approximately 350 words in length.

\section{Negative PTP Pilot}

A pilot study was conducted to determine the items that were used as the negative PTP items. A six-page summary of the most relevant trial information was constructed from the videotape (the summary was read by several raters and 
judged to contain all the important evidentiary information that was in the full trial). Participants $(N=22)$ read the summary of the trial, gave a rating of guilt, and then rated 50 fabricated pieces of evidence. Participants were asked to judge each item individually when deciding how that particular piece of information, if known in addition to what was in the summary, would affect their verdict. A 9 -point scale $(1=$ more likely guilty of manslaughter, $5=$ no effect, $9=$ more likely guilty of murder) was used to determine both the initial rating of guilt and how each additional item would influence the participant's verdict. Items that were significantly greater than $5, p<.05$, and could easily be worked into a news story were chosen as the negative PTP items (see Table 1). Negative items with lurid details were included in the emotional PTP article, whereas unemotional, extraevidentiary negative items were used for the factual PTP.

\section{Manipulation Check}

A second pilot study was conducted to insure that both the factual and emotional PTP articles produced significant bias toward the defendant's being guilty of murder compared to the control article. This was accomplished by randomly as-

Table 1. Factual and Emotional PTP Items

Type of PTP PTP Items

Emotional PTP 1. Kelly [the defendant] ran back to the bedroom and started to crush her dead mother's skull with a baseball bat.

2. Kelly would frequently flush her mom's heart medication down the toilet.

3. Kelly's mom came to Brad [the defendant's brother and victim's son] sobbing because Kelly had stabbed her in the left eye with the car keys.

4. Kelly had recurring dreams of chopping up her mother into little bits.

5. Kelly was restrained after she was found straddling her screaming brother while holding a knife to his throat.

6. The 911 operator reported that she could hear Kelly cackling in the background when Brad called in to report the stabbing.

7. Detectives reported finding journals of Kelly's that included detailed descriptions on how to dismember a victim's body.

8. Kelly's former cell mates reported that Kelly told them of the pleasure she experiences from seeing agony and pain in the eyes of her victims.

Factual PTP 1. Kelly fled the scene of the crime.

2. Gloria [the victim] had repeatedly stated that she was scared of Kelly.

3. A detective reported that Kelly had failed a lie detector test.

4. Kelly threatened to kill her mother.

5. Kelly had also told Brad [her brother] that their mother deserved to die.

6. Kelly had tried to kill a patient while she was in the hospital.

7. Kelly had tried to kill her brother Brad a year earlier.

8. Kelly told her [a friend] that she would fake being mentally ill to get away with her mother's murder. 
signing 114 participants to read one of the three articles and give a rating of guilt on a 9 -point scale $(1=$ more likely guilty of manslaughter, $5=$ undecided, $9=$ more likely guilty of murder). A one-way ANOVA yielded a significant difference in guilt ratings among the three articles, $F(2,111)=11.31, p<.001$. Scheffe's test revealed that ratings of guilt for emotional $(M=7.45)$ and factual $(M=7.45)$ PTP were significantly greater than for the control article $(M=5.39), p \mathrm{~s}<.05$, but not significantly different from each other.

Participants were also asked to give a rating of emotional arousal on a 9point scale $(1=$ not emotionally arousing, $9=$ highly emotionally arousing $)$. This was done to insure that the factual PTP and emotional PTP differed significantly in the level of emotional arousal they produced. There was a significant difference found among the three articles that participants read, $F(2,111)=8.31, p<.001$. Scheffe's test revealed that the factual $(M=6.55)$ PTP and the control article $(M=$ 6.29) were not rated as significantly different from each other in emotional arousal; however, both articles were rated as significantly less emotionally arousing than the emotional $(M=7.66)$ PTP $(p<.05)$. The videos were constructed from the written PTP after pilot testing was completed.

\section{Dependent Variables}

Participants were asked to fill out two different dependent measures of guilt. Participants first made a dichotomous judgment of whether the defendant was guilty of manslaughter or murder. Second, participants were asked to fill out a 4-point confidence rating $(1=$ not confident, $4=$ highly confident $)$ of their dichotomous judgment of guilt. These measures were combined into an 8-point continuous rating scale of guilt. Lower scores indicated high confidence in a manslaughter verdict, whereas higher scores indicated high confidence in a murder verdict. Thus, on the continuous rating scale of guilt, ratings of 1-4 concerned manslaughter verdicts ( 1 = highly confident-manslaughter, $4=$ not confident-manslaughter $)$, whereas ratings of 5-8 concerned murder verdicts $(5=$ not confident-murder, $8=$ highly confident-murder).

\section{Procedure}

Participants were run in groups of up to 15 people per session. The experiment was conducted in three phases: exposure to pretrial publicity, viewing the trial, and filling out the dependent measures. First, participants were randomly assigned to one of the six conditions. Participants were exposed to one of the three different PTP conditions, presented through either a video or a written medium. After exposure to the PTP, participants viewed the trial. Finally, participants in all conditions were asked to fill out the measures of guilt. All participants received pattern jury instructions instructing them on the relevant legal criteria and to make their decision based solely on the evidence presented at trial. The experiment lasted 
approximately $3 \mathrm{hr}$. At the end of the experiment, participants were debriefed and awarded their extra credit.

\section{RESULTS}

\section{Manipulation Check}

Manipulation checks were conducted to insure that participants were paying equal attention to the written and videotaped PTP. Participants were asked how closely they watched/read the video/article by circling a number on a 7-point scale $(1=$ not closely at all, 7 = very closely). There was no significant difference in the participants' reports of how closely they watched/read the PTP, $t(86)=.17$, $p>.05$, indicating that participants paid equal attention to the written $(M=5.47)$ and video $(M=5.46)$ PTP.

\section{Verdicts: Murder versus Manslaughter}

Logistic regression was used to analyze the dichotomous verdicts (shown for each condition in Table 2). There were three planned comparisons, two involving type of PTP (negative PTP vs. control, and factual vs. emotional PTP) and one involving PTP medium (video vs. written). As predicted, there was an effect of negative PTP on jurors' dichotomous judgments of guilt, $B=1.51, p<.005$. Participants were more likely to give a murder verdict when exposed to PTP $(73 \%$ murder verdicts) compared to participants in the control condition (39\% murder verdicts). However, verdicts for emotional PTP (80\% murder verdicts) were not significantly different from factual PTP (67\% murder verdicts), $B=.72, p=.23$. Finally, the dichotomous ratings did not replicate Ogloff and Vidmar's (1994) finding that PTP medium affected juror decision making (video PTP: 67\% murder verdicts; written PTP: 57\% murder verdicts), $B=.54, p=.25$.

Table 2. Percentage of Murder Verdicts and Mean Ratings of Guilt for Type of PTP and PTP Medium

\begin{tabular}{|c|c|c|c|c|c|c|c|c|}
\hline \multirow[b]{3}{*}{ PTP type } & \multicolumn{8}{|c|}{ PTP medium } \\
\hline & \multicolumn{4}{|c|}{ Video } & \multicolumn{4}{|c|}{ Written } \\
\hline & $(\%)$ & Mean & $S E$ & $N$ & $(\%)$ & Mean & $S E$ & $N$ \\
\hline Control & 47 & 3.80 & .74 & 15 & 31 & 3.61 & .77 & 13 \\
\hline Emotional & 87 & 6.53 & .55 & 15 & 73 & 5.87 & .63 & 15 \\
\hline Factual & 69 & 5.75 & .67 & 16 & 64 & 5.50 & .69 & 14 \\
\hline
\end{tabular}

Note. Ratings of 1-4 indicate a verdict of manslaughter, and ratings of 5-8 indicate a verdict of murder. More extreme numbers indicate greater confidence in the respective verdicts. (\%) $=$ Percentage of murder verdicts. 


\section{Continuous Ratings of Guilt}

The mean level of guilt for the different conditions can be seen in Table 2 . Planned comparisons were conducted for the effect of medium and the effect of PTP type on jurors' guilt ratings. The results are consistent with the dichotomous ratings. As predicted, participants exposed to some type of PTP reported significantly higher guilt ratings than participants exposed to the control article, $t(86)=3.71, p<.001, d=.80$ (Cohen, 1988). Guilt ratings for emotional PTP were not significantly different from guilt ratings for factual PTP, $t(58)=0.86, p=.20$, $d=.17$. Inconsistent with previous research (Ogloff \& Vidmar, 1994), there was not a significant effect of PTP medium on participants' guilt ratings, $t(86)=0.66$, $p=.26, d=.14$.

\section{DISCUSSION}

Jury simulation research has received considerable methodological criticism (e.g., Bray \& Kerr, 1982; Thompson, 1993). In order to understand the limits of generalizability, it is important to compare the different methodologies used in jury research to see if these different methods produce different results. There are a number of methodological implications in the present research for conducting simulation research on PTP, concerning the type of PTP and the PTP medium.

The present research had three objectives: First, we sought to replicate the general finding that negative PTP adversely affects mock jurors' predeliberation judgments compared to a control article. Second, emotional and factual PTP were compared directly to determine their effects on mock jurors' decisions relative to each other. Finally, the medium through which PTP was presented was examined to determine whether video PTP was more damaging than written PTP when the PTP was followed by a trial.

\section{PTP Affects Jurors' Decisions}

Participants were more likely to reach a murder verdict if they were exposed to negative PTP compared to a control article. The control condition in the present research was an article that contained the basic facts about the case. In a certain sense, participants were therefore still receiving pretrial publicity by being exposed to some of the facts of the case before trial. Future research including a "no article" control condition would allow researchers to examine varying levels of exposure to information about a case and its effects on juror decision making. Nonetheless, the present finding supports the bulk of the literature indicating that negative (compared to neutral) PTP has a deleterious effect (Costantini \& King, 1980; Hoiberg \& Stires, 1973; Kramer et al., 1990; Moran \& Cutler, 1991; Ogloff \& Vidmar, 1994; Otto et al., 1994; Padawer-Singer \& Barton, 1975; Simon \& Eimermann, 1971; Sue et al., 1974, 1975; Tans \& Chaffee, 1966), while also extending the effect's generality. The cases that have been used in previous PTP studies have had participants make 
decisions on whether the defendant is guilty or not guilty (e.g., Kramer et al., 1990; Ogloff \& Vidmar, 1994; Otto et al., 1994). In the present experiment, the issue at trial was not whether or not the defendant was guilty of a particular act, but rather of which of two acts the defendant was guilty (i.e., murder vs. manslaughter). To our knowledge, this is the first study that has shown that PTP can affect jurors' decisions in the determination of which of two acts was committed. Thus, the effect of PTP has been generalized to a new context.

\section{Factual versus Emotional PTP}

There was insufficient evidence to conclude that emotional PTP was significantly more prejudicial than factual PTP. This provides evidence that if the amount and duration of PTP as well as its degree of bias (i.e., functional equivalence) are held constant, then the effect of PTP is not significantly different for emotional and factual PTP. This was true even though the emotional PTP article was perceived as significantly more arousing than the factual PTP article. It is quite possible that the emotional arousal played a significant role in why the emotional PTP was functionally equivalent-with regard to its effect on participants' perception of the defendant's guilt-to the factual PTP, despite being factually less informative. Similarly, it could also be the case that the emotional arousal had nothing to do with the reason why the emotional PTP was functionally equivalent to the factual PTP; that is, the failure to find a significant difference between the two articles could be explained by the fact that the two articles contained different items of information. However, even though the information in the articles was incongruent, the emotional PTP was equivalent to the factual PTP in the sense that both types of PTP were legally irrelevant, had the same amount and duration, and produced the same degree of bias. Future research should address whether emotionally arousing PTP can affect jurors' ultimate guilt judgments without in some way being perceived as relevant to the issue of the defendant's guilt, as well as whether the same information could somehow be manipulated so as to vary its emotionality while holding its functional impact constant.

Although previous research has addressed the influence of both emotional and factual PTP (Hoiberg \& Stires, 1973; Kramer \& Kerr, 1989; Kramer et al., 1990), the present study was the first attempt to compare their effects on mock jurors' judgments directly. The results of the present research support previous findings that both types of PTP can influence participants' judgments compared to neutral information (e.g., Kramer \& Kerr, 1989; but see Hoiberg \& Stires, 1973). However, we found no difference between factual and emotional PTP. Therefore, considering the previous research on emotional and factual PTP (e.g., Kramer \& Kerr, 1989; Kramer et al., 1990), it may not have been the emotional arousal per se causing the effects of emotional PTP, but the information that the PTP contained. More research is needed to understand exactly how emotional, nonfactual PTP exerts its effects. 
There are limits to the conclusions we can draw about the emotional versus factual PTP distinction. Although the emotional PTP used in this study was rated as emotionally more arousing than the factual PTP, this statistically significant difference does not provide any evidence on how arousing the emotional PTP was in general. For example, graphic footage of violent crimes is likely to elicit more emotional arousal than a news reporter merely describing the event.

\section{PTP Medium}

The results found by Ogloff and Vidmar (1994) were not replicated. Video PTP did not produce higher guilt ratings than written PTP when the content was held constant and participants were also presented with a trial. Although videotaped information is more persuasive than written information in some contexts (Chaiken \& Eagly, 1976, 1983), this effect was not obtained in the present PTP manipulation. One explanation of this discrepancy could be the complex nature of the PTP. Chaiken and Eagly $(1976,1983)$ have found that the advantage for videotaped information is reduced when the message is hard to understand. However, Ogloff and Vidmar (1994) also used relatively involved PTP concerning a complex trial.

Is there any way to reconcile Ogloff and Vidmar's (1994) findings with the present research? Some research using individual juror data has shown that presentation of a trial weakens or eliminates the effect of PTP (Otto et al., 1994; Kramer et al., 1990). For example, Otto et al. (1994) found that participants' ratings of guilt were weaker after they had viewed the trial compared to their pretrial ratings. Similarly, Kramer et al. (1990) found that individual juror ratings revealed no effect of PTP after participants had been exposed to a trial. Our failure to replicate the difference between video and written PTP found by Ogloff and Vidmar (1994) suggests that presenting a trial could possibly mitigate the effect of PTP medium in the same way that it appears to mitigate the effect of PTP in general (Otto et al., 1994).

Alternatively, it is possible that in controlling for everything but medium, the present experiment eliminated the effect of video over written PTP. It is impossible to disentangle whether the null effect of video versus written PTP is due to our manipulation of the medium, exposure to a trial, or a combination of both. Future research is needed to address this issue. However, in investigating the potential effects of variations in PTP, it is important to assess any effects in the more forensically valid situation in which a trial actually follows the pretrial publicity. The question of presentation mode is an important one because if one mode of PTP affected jurors' decisions, but another mode did not, only jurors who had been exposed to the "damaging mode" would be candidates for exclusion during voir dire. Thus, this type of research merits further investigation because of the policy implications of the free speech/fair trial debate (Carroll et al., 1986; Fulero, 1987; Linz \& Penrod, 1992), as well as the methodological implications that arise from presenting experimental materials in different manners. 


\section{CONCLUSIONS}

The present research examined two methodological issues within the PTP literature. There were three main findings: First, there was an effect of PTP on individual mock jurors' guilt judgments. Second, no significant difference was found between factual and emotional PTP when controlling for the PTP's functional impact. Third, no significant difference was found between video and written PTP when controlling for the message's content and showing a trial.

A consideration of the methodological issues involved in conducting research on PTP is necessary to provide the foundation that researchers need to present formal guidelines to the judicial system on the effects of PTP (Carroll et al., 1986; Fulero, 1987). If a body of realistic and reliable evidence can be accumulated on the effects of PTP on juror and jury decision making, then social scientists will be in a better position to inform the courts on guidelines for handling PTP (Carroll et al., 1986).

\section{ACKNOWLEDGMENTS}

This research was conducted as part of a master's thesis by the first author under the supervision of the second author. It was presented at the 7th annual Southwest Conference on Cognition in Austin, Texas. We thank Jennifer Lindsey and Roy Wilson for their help in constructing the PTP materials, and Brittan Barker and Elizabeth Nguyen for their assistance in various phases of the project. We are also very grateful to Steven Penrod for providing a copy of the videotaped trial.

\section{REFERENCES}

Bray, R. M., \& Kerr, N. L. (1982). Methodological consideration in the study of the psychology of the courtroom. In N. Kerr \& R. Bray (Eds.) The psychology of the courtroom (pp. 287-323). New York: Academic Press.

Carroll, J. S., Kerr, N. L., Alfini, J. J., Weaver, F. M., MacCoun, R. J., \& Feldman, V. (1986). Free press and fair trial: The role of behavioral research. Law and Human Behavior, 10, 187-201.

Chaiken, S., \& Eagly, A. H. (1976). Communication modality as a determinant of message persuasiveness and message comprehensibility. Journal of Personality and Social Psychology, 34, 605-614.

Chaiken, S., \& Eagly, A. H. (1983). Communication modality as a determinant of persuasion: The role of communicator salience. Journal of Personality and Social Psychology, 45, 241-256.

Cohen, J. (1988). Statistical power analysis for the behavioral sciences (2nd ed.). Hillsdale, NJ: Erlbaum.

Costantini, E., \& King, J. (1980). The partial juror: Correlates and causes of prejudgment. Law and Society Review, 15, 9-40.

Davis, R. W. (1986). Pretrial publicity, the timing of the trial, and mock jurors' decision processes. Journal of Applied Social Psychology, 16, 590-607.

Fulero, S. M. (1987). The role of behavioral research in the free press/fair trial controversy. Law and Human Behavior, 11, 259-264.

Hoiberg, B. C., \& Stires, L. K. (1973). The effect of several types of pretrial publicity on the guilt attributions of simulated jurors. Journal of Applied Social Psychology, 3, 267-275.

Kramer, G. P., \& Kerr, N. L. (1989). Laboratory simulation and bias in the study of juror behavior. Law and Human Behavior, 13, 89-99. 
Kramer, G. P., Kerr, N. L., \& Carroll, J. S. (1990). Pretrial publicity, judicial remedies, and jury bias. Law and Human Behavior, 14, 409-438.

Linz, D., \& Penrod, S. (1992). Exploring the first and sixth amendments: Pretrial publicity and jury decision making. In D. K. Kagehiro \& W. S. Laufer (Eds.), Handbook of psychology and law (pp. 3-20). New York: Springer-Verlag.

Moran, G., \& Cutler, B. L. (1991). The prejudicial impact of pretrial publicity. Journal of Applied Social Psychology, 21, 345-367.

Moran, G., \& Cutler, B. L. (1997). Bogus publicity items and the contingency between awareness and media-induced pretrial prejudice. Law and Human Behavior, 21, 339-334.

Ogloff, J. R. P., \& Vidmar, N. (1994). The impact of pretrial publicity on jurors: A study to compare the relative effects of television and print media in a child sex abuse case. Law and Human Behavior, 18, 507-525.

Otto, A. L., Penrod, S. D., \& Dexter, H. R. (1994). The biasing impact of pretrial publicity on juror judgments. Law and Human Behavior, 18, 453-469.

Padawer-Singer, A. M., \& Barton, A. H. (1975). The impact of pretrial publicity on jurors' verdicts. In R. J. Simon (Ed.), The jury system in America: A critical overview (pp. 125-139). Beverly Hills, CA: Sage.

Padawer-Singer, A,, Singer, A,, \& Singer R. (1977). Legal and social-psychological research in the effects of pretrial publicity on juries, numerical makeup of juries, non-unanimous verdict requirements. Law and Psychology Review, 3, 71-79.

Simon, R. J. (1980). The jury: Its role in American society. Lexington, MA: Lexington Books.

Simon, R. J., \& Eimermann, T. (1971). The jury finds not guilty: Another look at media influence on the jury. Journalism Quarterly, 48, 343-344.

Sue, S., Smith, R. E., \& Gilbert, R. (1974). Biasing effect of pretrial publicity on judicial decisions. Journal of Criminal Justice, 2, 163-1 71.

Sue, S., Smith, R. E., \& Pedroza, G. (1975). Authoritarianism, pretrial publicity, and awareness of bias in simulated jurors. Psychological Reports, 37, 1299-1302.

Tans, M. D., \& Chaffee, S. H. (1966). Pretrial publicity and juror prejudice. Journalism Quarterly, 43, 647-654.

Thompson, W. C. (1993). Research on jury decision making: The state of the science. In N. J. Castellan (Ed.), Individual and group decision making: Current issues (pp. 203-218). Hillsdale, NJ: Erlbaum. 